\title{
Preface
}

\section{Selected papers from ISEF-2017 Conference}

Since its very beginning in Poland in the year 1974, ISEF conference is a meeting point of scientists promoting different cultures and knowledge. In fact, the international community behind the conference - which grew up substantially in the years - joins academic and industrial researchers, working in the broad area of applied electromagnetic. Here, a collection of 25 papers, presented at the conference and selected after a peer-review procedure, covers the main subjects of interest for the ISEF community. The conference took place in Lodz (Poland) from 14th through 16th September 2017.

A group of papers is focused on optimal design methods with application in electromechanics; more often than not, the subject of design uncertainty is considered in a systematic way within the optimal design procedure. In general, gradient-free methods attempt to approximate the minimum of one or more objective functions, subject to various constraints and simultaneously dependent on a set of design variables. Applications range from classical power devices, like induction or permanent-magnet motors, to micro-electro-mechanical systems.

An emerging new tendency is multi-physics - or coupled field - approach to modelling and simulation. Often the solution of field problems in single-physics domains is no longer sufficient to cope with the challenge of model complexity, and coupling different field problems is mandatory. Vibration analysis in permanent-magnet motors or thermal effect simulations of induction motors are just a few examples.

Modelling innovative materials is the subject of another group of papers, where designing nanostructures for EMC, or identifying the normal permeability of electrical steel sheets is the main focus.

The research in traditional fields like e.g. electromechanics moves towards new applications: small textile antennas for biomedical applications or energy transfer by means of a radiated electromagnetic field are just examples of innovative subjects covered by a few papers.

Almost always, field analysis is the common ground upon which models of specific devices are developed: in fact, field-based models are especially suited to predict the behaviour of devices exhibiting complex shapes and multi-property materials, or to synthesize new devices characterized by better performance or lower cost than existing prototypes. In general, the underlying message is that university and industry should cooperate in a closer way to dominate the challenges of product innovation.

Last, not least, the conference is also the meeting point of different generations of scientists, where the reputation of seniors and the enthusiasm of juniors combine together promoting a mutual benefit.

$$
\begin{array}{r}
\text { Sławomir Wiak }{ }^{2}, \text { Paolo Di Barba }{ }^{1}, \text { Krzysztof Komeza }^{2} \\
{ }^{1} \text { University of Pavia, Pavia, Italy } \\
{ }^{2} \text { Łódź University of Technology, Łódź, Poland }
\end{array}
$$

IJAEM Guest Editors 